Volume 8, No. 7, July - August 2017

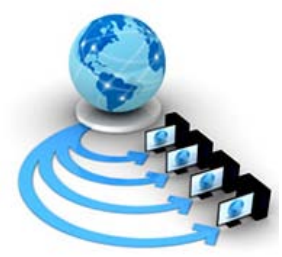

International Journal of Advanced Research in Computer Science

RESEARCH PAPER

\author{
Available Online at www.ijarcs.info
}

\title{
AN EFFICIENT BRAIN TUMOUR DETECTION SYSTEM BASED ON SEGMENTATION TECHNIQUE FOR MRI BRAIN IMAGES
}

\author{
Surabhi Kaushal \\ Under the Guidance of: Assit. Prof. Er. Beant kaur \\ Deptt. Of ECE \\ Punjabi University,Patiala,India
}

\begin{abstract}
In this work, we have proposed an efficient brain tumour detection system based on the segmentation technique for MRI brain images, which can detect tumour and trace the position of tumour in the MRI brain images. To detect the tumour region, segmentation method is used on the basis of threshold and it exactly detects the tumour region with better accuracy. In the proposed work, some pre-processing steps (resizing, binarization and morphological operations) are used for detection of tumour region in MRI brain images. It is being observed that the experimental results of the proposed brain tumour detection system gives better result in comparison to other existing techniques. This study introduces an efficient detection of brain tumour from MRI brain images based on the segmentation of gray matter and white matter. The purpose of this segmentation algorithm is to label tumour objects within the MRI brain image and locate their region based on the white matter. The tumour is extracted from the MRI brain images and its exact position is also determined to classify the volume of tumour. By experimental analysis of various parameters such as PSNR, MSE and volume or percentage of tumour, we have observed that the accuracy of proposed system is more that $90 \%$ and it is better than the previous work.
\end{abstract}

Keywords: Brain tumour, MRI images, Segmentation, Threshold, PSNR, MRI

\section{INTRODUCTION}

A brain tumour is a compilation of the abnormal cells in brain [1]. A tumour can results to cancer, which is the main reason of death and accountable for around 13\% of every deaths world-wide. Cancer occurrence rate is rising at an alarming rate in the world. Therefore, tumour detection is significant in previous stages. Huge knowledge and knowledge on radiology are necessary for precise tumour detection in medical imaging. MRI (magnetic resonance imaging) is considered as the most flexible of the modalities of diagnostic imaging with the capability to characterize an extensive range of parameters in the living subject and gives fine spatial resolution. Identification of brain tumour form MRI is consisted of different stages [2]. Segmentation is termed to be a significant but tough step for the classification of medical imaging and its analysis. Therefore, it is important that segmentation of the MRI images should be done precisely before asking the processor for accurate diagnosis.

This research has focussed on verifying the accuracy and efficiency of brain tumour detection via segmentation technique for MRI images of brain [3].

\subsection{Brain}

Usually, the human brain is consisted of three major parts for controlling the unlike activity as defined below [4]:

i. Cerebrum: It controls the diverse activities like thinking, learning, emotions, speech, and reading, problem solving and writing. Cerebrum is characterized in right and left cerebral hemispheres.

ii. Cerebellum: It controls different movements, balance, standing, and complex actions.

iii. Brain Stem: The spinal cord connected with the brain by the brain stem. The brain stem executes the control action and controls body temperature, blood pressure and controls different vital functions

Below are the basic symptoms for brain tumour. Though, human being might face the symptoms differently and may vary on the basis of location and size of the tumour [5].

- Headache

- Vomiting (usually in the morning)

- Nausea

- Personality changes

- Irritability

- Drowsiness

- DepressionDecreased cardiac and respiratory function

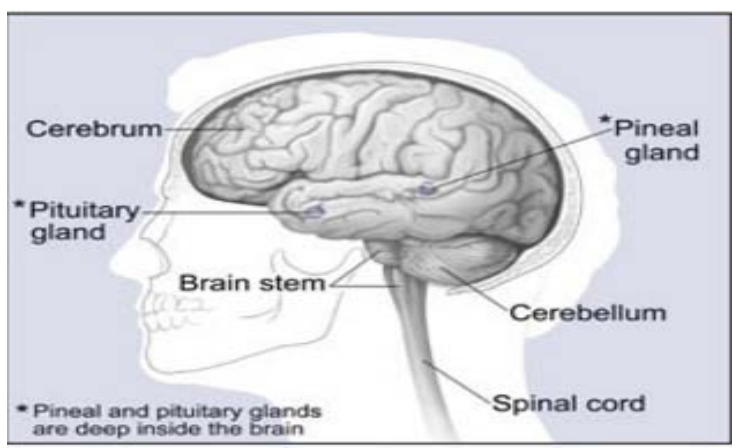

Figure 1: Human Brain Major parts Tumour

"Tumour" is a synonym used for a word "neoplasm" which is defined as an irregular growth of cells. Tumour is totally dissimilar from the cancer. The types of tumour are explained below [6]:

i. Benign: It is a tumour which does not enlarges in an unexpected manner. It doesn’t affect its neighbouring healthy tissues and also does not expand to nonadjacent tissues. Moles are considered as the general example of benign tumours. 
ii. Pre-Malignant: This tumour is a stage of pre-cancerous which is treated as a disease, which if not correctly treated it might lead to cancer.

iii. Malignant: It is the kind of tumour which became worse with the time span and eventually results in the death of a human being. It is generally a medical word that defines a serious progressing disease. It is normally utilized for the explanation of cancer.

\subsection{MRI (Magnetic Resonance Imaging)}

MRI is generally utilized in the biomedical for detecting and visualizing finer particulars in the body's internal structure [7]. This method is generally utilized for detecting the differences in the tissues that has a far enhanced method as compared to CT (computed tomography). Therefore, this defines this method a special one for the detection of brain tumour and cancerous images [8]. MRI consists of valuables in the surgical treatment of brain by granting the regions by means of significant brain functions to be located. The surgeon may keep away from damaging the regions when great disease or dysfunctional tissues are removed [9].

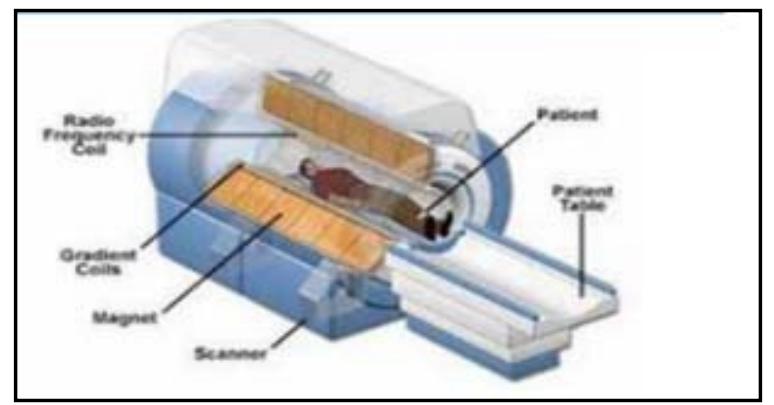

Figure 2: MRI Scanner

\section{CLASSIFICATION OF BRAIN TUMOUR DETECTION}

This section defines the various classifications used for the detection of brain tumour by steps, namely, Pre-processing, Feature Extraction, Segmentation, Post-processing and son on that are utilized for determining the tumour area of MRI Images [10]. Below figure is defining the feature extraction basic structure via DIP (Digital Image Processing).

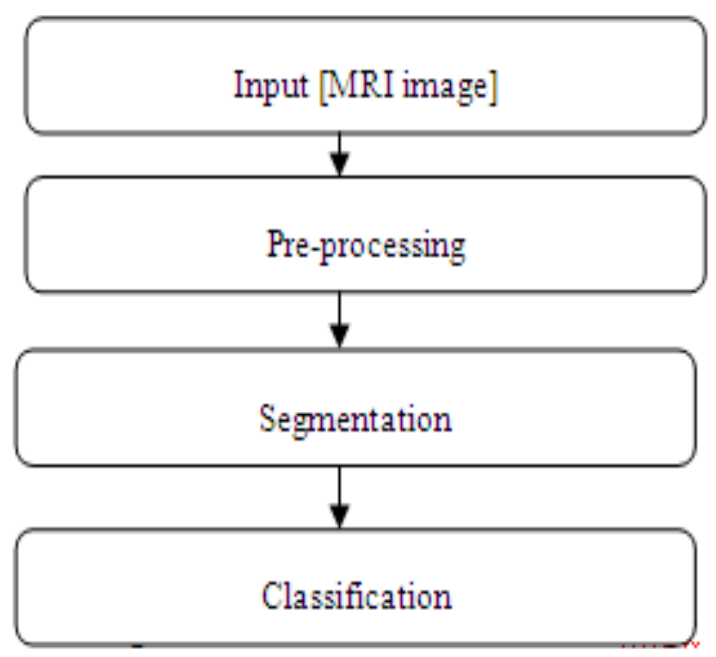

Figure 3: Feature Extraction block diagr i. Pre-processing
In pre-processing stage, the captured image is converted into gray scale and the size of the image also reduced according to the requirement. In this stage, only the irrelevant data is removed so the image became a better to operate [11]. It is defined as the number of operations executed on the scanned input MRI brain image. It significantly improves the image rendering appropriate for segmentation of tumour. The aim of pre processing is improving the results of the segmentation. Normally, smoothing, noise filtering and normalization need to be executed in this process. The preprocessing also explains the compact pattern representation. The binarization procedure transforms a gray scale image in binary image which helps to segment image based on the threshold value [12].

\section{ii. Segmentation}

In this work segmentation technique is necessary for the gray matter mask and white matter mask extraction based on the binary image. The binary image consists of information on the basis of shape and position of an object of the mask image [13]. The benefit of binary image is that it lessens the image complexity and it simplifies the procedure. The methods of threshold detection are: Optimal thresholding, adaptive thresholding, mixture thresholding and P-tile thresholding and in this work optimal thresholding is used to segment the tumour region from the MRI brain image.

\section{iii. Classification}

To detect the tumour region from the MRI brain images, the classification steps plays a big and important role [14]. For classifying the tumour region from the brain images, we have created a mask of gray and white matter based on the binary image. In the binary image, data is represented in the form of 0 and 1 , and we have set threshold to create gray matter and white matter mask. In the MRI image, the affected part represents by the white matter and on the basis of white matter, we have classified the tumour region and their volume [15].

\section{RELATED WORK}

This section explains the glance of existing techniques by number of authors in the field of digital image processing for the detection of brain tumour.

S. Murugavalli and V. Rajamani have reviewed the MRI image performance for execution time, weight vector, and tumour pixels compared and detected the outcomes with the related ones. The authors have achieved an enhanced value of detected tumour pixels as compare to some another segmentation methods. The authors has achieved the weight vector value with the neuro fuzzy is $(6 \times 6)$ with the more input features. The author has also studied the weight vector value with the number of tumour cells with the execution time with diverse technique of distance classifiers. The variation of growth rate for the similar patient is also undertaken.

Marcel Prastawa et al. has defined a network for segmentation of automatic brain tumour for MRI. The edema detection is executed simultaneously by tumour segmentation because the knowledge of the extent of edema 
is significant for planning, diagnosis and treatment. It has been confirmed from the collaborating clinic that is the most relevant feature because the edema region might need secondary analysis with the treatment after the focus to the tumour region. The proposed technique has utilized a concept for detecting the difference by means of normal and utilizes non-parametric estimation for the distribution as compare to existed mixture Gaussian models. The method has also utilizes another features because of the shape, intensity and location of edema.

T. Logeswari and M. Karnan has defined segmentation method by two phases. In the initial phase, the MRI brain image is taken from patients' database. In that prospect, noise and artifact are removed after that HSom is executed for image segmentation. The HSom is the addition of the conventional SOM (Self organizing map) utilized for classifying the image row by row. In the lowest level of weight vector, an enhanced value of tumour pixels, computation speed is obtained by the HSom with vector quantization.

Rajeev Ratan and Sanjay Sharma has shown that the Watershed Segmentation successfully segment a tumour providing a parameters that are set correctly in MATLAB platform. The performance of watershed Segmentation algorithm is better for the cases in which the intensity level difference among the tumour and non-tumour segments being higher. It may also segment non-homogenous tumours giving the non homogeneity is within the tumour region. The research has proves that the proposed methods has general purpose segmentation tools within the medical imaging that could be utilized for automatic segmentation for brain tumours. The segmentation quality is same as manual segmentation and has speed up segmentation in the operative imaging.

Sudipta Roy and Samir Bandyopadhyay has presented interactive segmentation techniques that enable the users to efficiently and quickly segment the tumours in MRI of brain. The author has proposed a novel method with the area of the region and edge information utilizes a kind of prior information with its symmetry analysis being more consistent in pathological cases. Its application to different datasets with different intensities, tumours sizes, and locations has shown that it may automatically detect and segments very diverse brain tumours types with a better quality.

N. Nandha Gopal and Dr. M. Karnan has designed an intelligent system for diagnosing brain tumour via MRI by utilizing algorithms of image processing clustering like Fuzzy C Means with intelligent optimization tools, like GA (Genetic Algorithm), PSO (Particle Swarm Optimization). The tumour detection is being performed in two stages, namely, Enhancement and Pre-processing in the initial phase and classification with the segmentation in the next phase.
Khan M. Iftekharuddin et al. has find the effectiveness of fusing two new texture features with the intensity in multimodal MRI (Magnetic resonance images) for segmentation and classification of pediatric brain tumour. The author has also exploited three MR image modalities like T1 (gadolinium-enhanced), T2 with FLAIR (FLuidAttenuated Inversion-Recovery) correspondinlgly. The authors have conducted a multi-layer FFNN (Feedforward neural network) by automated Bayesian regularization for classifying the tumour regions from non-tumour regions. The ROC (Receiver Operating Characteristic) curves are produced for evaluating the performance of tumour classification The ROC has suggested that at a threshold value of 0.7 , the TPF (True Positive Fraction) values from $75 \%$ to $100 \%$ for diverse patients, with the average value of $90 \%$.

T.Logeswari and M.Karnan has reviewed MRI image performance for execution time, weight vector and tumour pixels detected. The authors have defined different methods in medical image processing. The author has also discussed needs and properties of brain tumour detection techniques. This research is utilized for providing more information for the detection and segmentation of brain tumour.

\section{SIMULATION MODEL}

To verify the efficiency and accuracy of proposed brain tumour detection using the segmentation technique for the brain MRI images, we have performed several experiments with this procedure on several brain MRI images. In proposed work, several steps have been used to detect the tumour region and the methodology of proposed work is given below:

Step 1: Design and develop a proper GUI of proposed brain tumour detection using the segmentation technique for the brain MRI images.

Step 2: Apply pre-processing on uploaded brain MRI image. In this phase, image is enhanced in the way that finer details are improved and image is being enhanced so the post processing can be applied easily and the accuracy can be maximum.

Step 3: A code is being developed for the brain MRI image segmentation technique to segment the tumour region. Threshold based segmentation is one of the simplest segmentation methods. The input gray scale image is converted into a binary format. The method is based on a threshold value for converting the gray scale image into a binary image format. The main logic is the selection of a threshold value.

Step4: After the detection of tumour from the brain MRI image, we have computed the performance metrics like MSE, PSNR, and measure tissue volume to measure growth of tumour. 


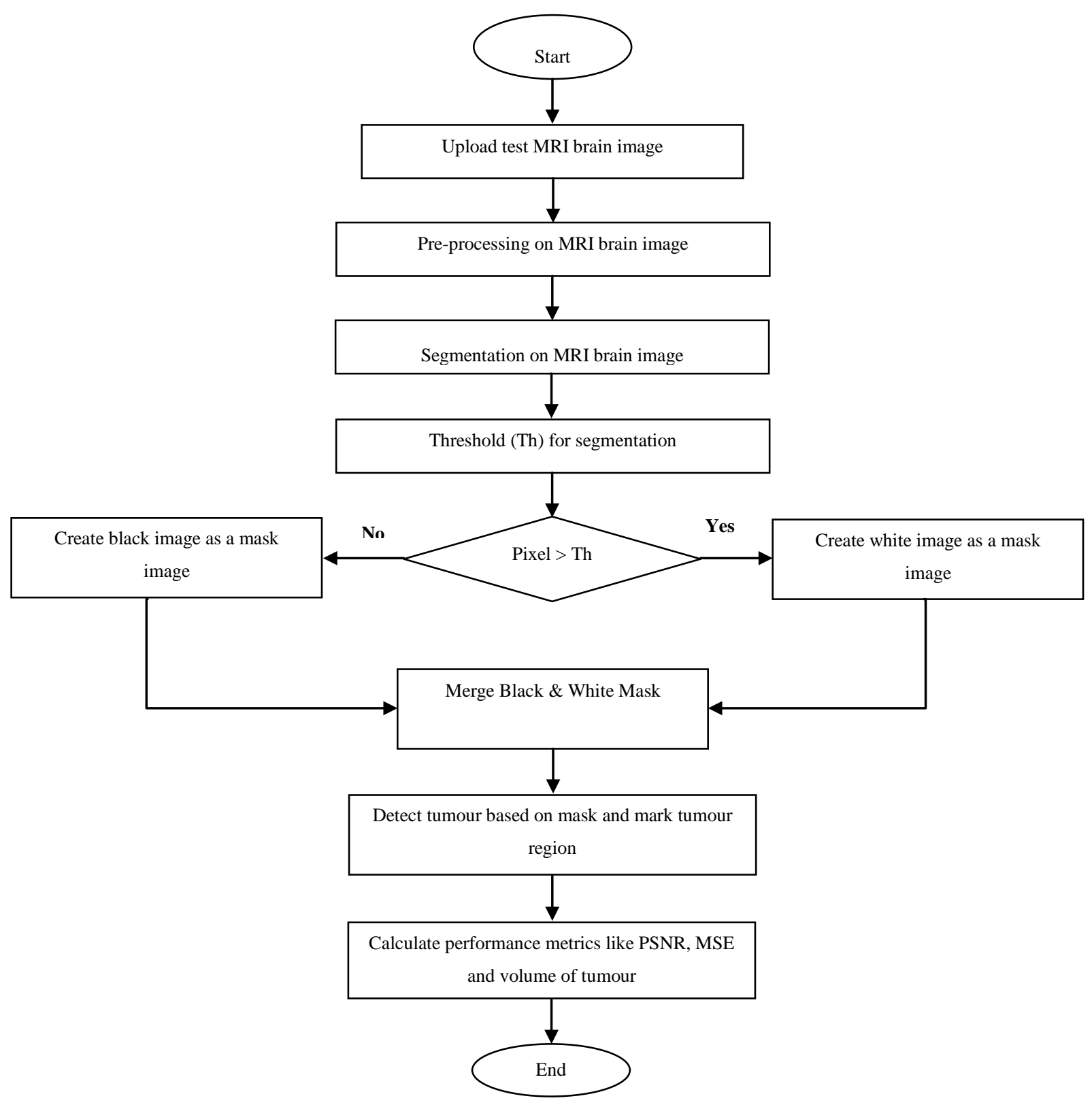

Figure 4: Proposed work Flowchart

\section{SIMULATION RESULTS}

This section explains the results obtained after the

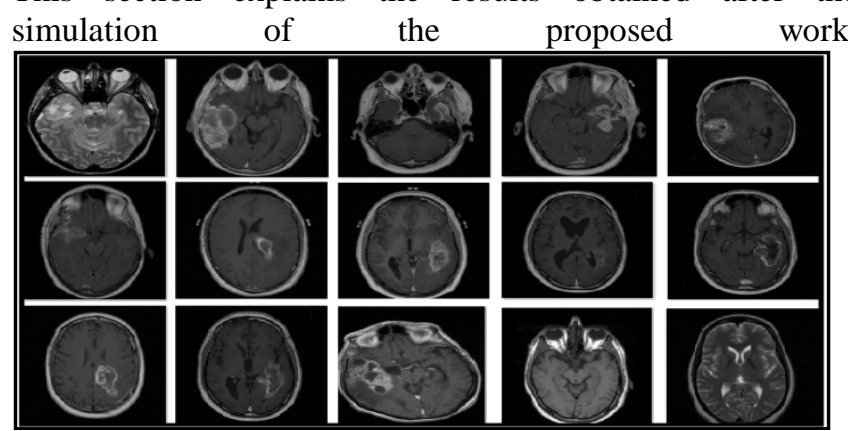

Figure 5: MRI Database of Brain
Above figure represents the used database in the proposed Brain Tumour Detection System using MRI based on the segmentation technique. The format of images in the database is BIM without any type of compression. In this dataset, the tissue structure and anatomical parts of the brain are described to understand the purpose of this study. The MRI brain image is composed of two types, namely gray matter and white matter. Gray matter is made of neuronal and White matter fibers consisted of many eliminated axons which connect the cerebral cortex with other brain regions. On the basis of Gray matter and White matter the tumour has been detected from MRI. 


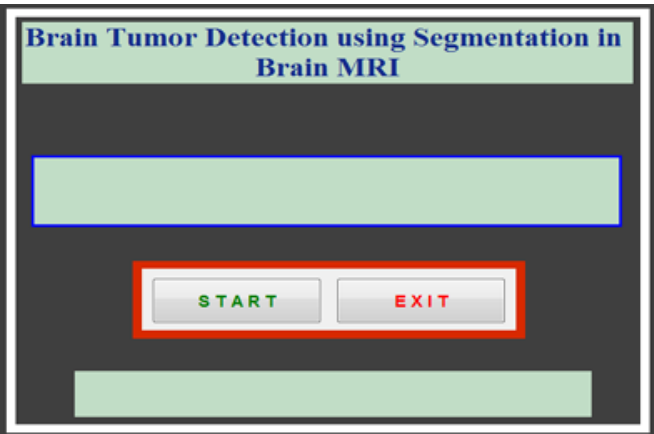

Figure 6: Title window of proposed work

Above figure show the main figure window of proposed work. Main figure window is the title page of proposed work and there are two options, $1^{\text {st }}$ is START and $2^{\text {nd }}$ is EXIT. If we press the START, then working figure window being displayed and if we press EXIT, then the model is being closed.

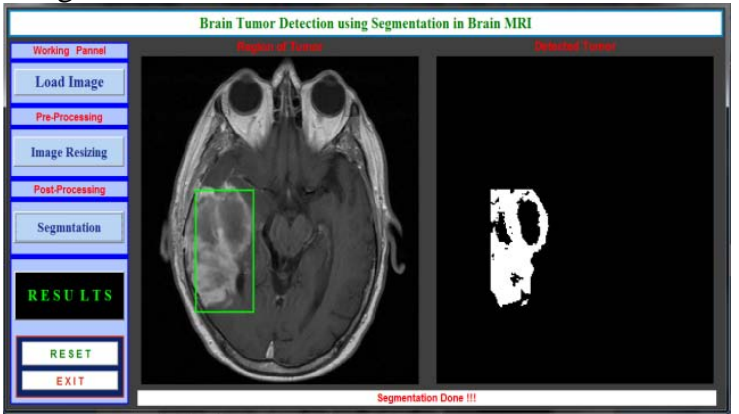

Figure 7: Segmentation and Detection of tumour

The above figure is showing the locating and detecting of tumour. In the first window, the region of tumour is being detected and after the detection, the detected region is located in the second window.

Table1: Parameters of proposed work

\begin{tabular}{|l|l|l|}
\hline PSNR & MSE & $\begin{array}{l}\text { Tumour } \\
\text { Percentage }\end{array}$ \\
\hline 42.41 & 3.76 & 46 \\
\hline 40.58 & 2.87 & 53 \\
\hline 41.98 & 4.58 & 51 \\
\hline 43.55 & 3.25 & 67 \\
\hline 44.96 & 4.89 & 72 \\
\hline
\end{tabular}

The above table is defining the values of different parameters obtained afetr the simulation of the proposed work. Parameters, namely, PSNR(Peak signal to noise ratio), MSE (Mean Square Error) and the percentage of tumour are considered for calculating the performance of the proposed work

\section{PSNR}

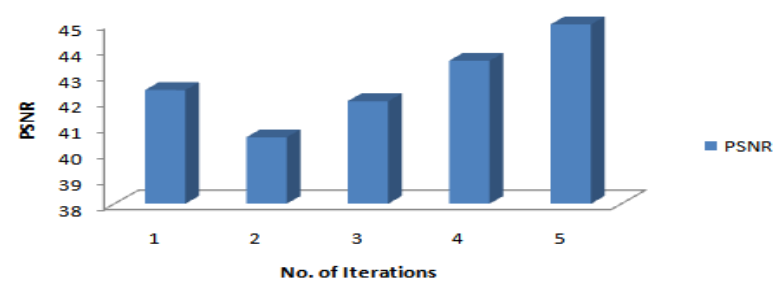

Figure 8: PSNR (Peak Signal to Noise Ratio)
The above figure 8 is showing a graph of PSNR. In the above graph, the blue color is increment of PSNR. X-axis is showing the number of iterations and Y-axis is showing the values obtained of PSNR. The average of PSNR obtained is 42.696 .

\section{MSE}

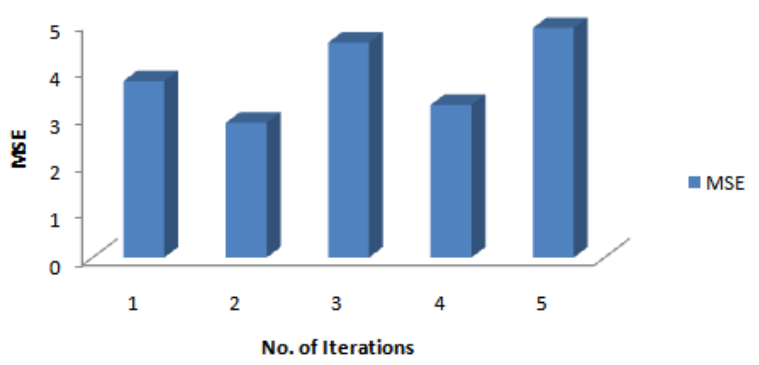

Figure 9: MSE (Mean Square Error)

The above figure is showing the graph of MSE. As depicted, the $\mathrm{X}$-axis is depicting the number of iterations and Y-axis is depicting the values obtained after the simulation of MSE. The average value of MSE obtained is 3.87.

Tumour Percentage

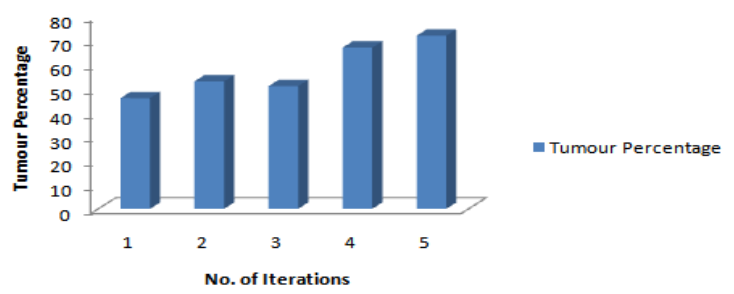

Figure 10: Tumour Percentage

The percentage of tumour is shown in the above figure in the form of graph. The $\mathrm{X}$-axis is depicting the Number of iterations and Y-axis is depicting the tumour percentage. It is being concluded that the tumour percentage is increasing simultaneously. The average percentage of tumour is 57.8.

\section{CONCLUSION}

In this proposed brain tumour detection system work, we have acquired MRI brain image to perform a series of operations and to enhance the quality of the image, we have applied pre-processing on image. After that, to segment the tumour region from the MRI brain image, threshold based segmentation technique is being applied. This segmentation algorithm is able to segment tumours clearly and able to outline the region of the tumour. The proposed segmentation algorithm was experimented with MRI brain images of human for detecting and locating tumour in the images. So, new concept based on the threshold can be used to improve the correctness of segmentation algorithms. By experimental analysis of proposed work, we have observed that the system accuracy is more than $90 \%$ to classify the tumour region from the MRI brain images.

In the future work, optimization of tumour region can be enhanced using the optimization algorithms to calculate the area and the thinness of the tumour present in the MRI. Feature extraction algorithms can also be used to classify the tumour region more accurately so that the efficiency of tumour detection system can be improved. 


\section{REFERENCES}

[1]. Kharrat A, Benamrane N, Messaoud MB, Abid M. Detection of brain tumour in medical images. InSignals, Circuits and Systems (SCS), 2009 3rd International Conference on 2009 Nov 6 (pp. 1-6). IEEE.

[2]. Murugavalli S, Rajamani V. An Improved Implementation of Brain Tumour Detection Using Segmentation Based on Neuro Fuzzy Technique 1.

[3]. Logeswari T, Karnan M. An improved implementation of brain tumour detection using segmentation based on soft computing. Journal of Cancer Research and Experimental Oncology. 2009 Mar 31;2(1):006-14.

[4]. Wu MN, Lin CC, Chang CC. Brain tumour detection using color-based k-means clustering segmentation. InIntelligent Information Hiding and Multimedia Signal Processing, 2007. IIHMSP 2007. Third International Conference on 2007 Nov 26 (Vol. 2, pp. 245-250). IEEE.

[5]. Prastawa M, Bullitt E, Ho S, Gerig G. A brain tumour segmentation framework based on outlier detection. Medical image analysis. 2004 Sep 30;8(3):275-83.

[6]. Iftekharuddin KM, Zheng J, Islam MA, Ogg RJ. Fractal-based brain tumour detection in multimodal MRI. Applied Mathematics and Computation. 2009 Jan 1;207(1):23-41.

[7]. Gondal AH, Khan MN. A review of fully automated techniques for brain tumour detection from MR images. International Journal of Modern Education and Computer Science. 2013 Feb 1;5(2):55.

[8]. Ratan R, Sharma S, Sharma SK. Brain tumour detection based on multi-parameter MRI image analysis. ICGST-GVIP Journal. 2009 Jun;9(3):9-17.

[9]. Logeswari T, Karnan M. An enhanced implementation of brain tumour detection using segmentation based on soft computing. InSignal Acquisition and Processing, 2010. ICSAP'10. International Conference on 2010 Feb 9 (pp. 243247). IEEE.

[10].Gopal NN, Karnan M. Diagnose brain tumour through MRI using image processing clustering algorithms such as Fuzzy C Means along with intelligent optimization techniques. InComputational Intelligence and Computing Research (ICCIC), 2010 IEEE International Conference on 2010 Dec 28 (pp. 1-4). IEEE.

[11].Roy S, Bandyopadhyay SK. Detection and Quantification of Brain Tumour from MRI of Brain and it's Symmetric Analysis. International Journal of Information and Communication Technology Research. 2012 Jun;2(6).

[12].Natarajan P, Krishnan N, Kenkre NS, Nancy S, Singh BP. Tumour detection using threshold operation in MRI brain images. InComputational Intelligence \& Computing Research (ICCIC), 2012 IEEE International Conference on 2012 Dec 18 (pp. 1-4). IEEE.

[13].Moffat BA, Chenevert TL, Lawrence TS, Meyer CR, Johnson TD, Dong Q, Tsien C, Mukherji S, Quint DJ, Gebarski SS, Robertson PL. Functional diffusion map: a noninvasive MRI biomarker for early stratification of clinical brain tumour response. Proceedings of the National Academy of Sciences of the United States of America. 2005 Apr 12;102(15):5524-9.

[14].Vijay J, Subhashini J. An efficient brain tumour detection methodology using K-means clustering algoriftnn. InCommunications and Signal Processing (ICCSP), 2013 International Conference on 2013 Apr 3 (pp. 653-657). IEEE.

[15]. Bhattacharyya D, Kim TH. Brain tumour detection using MRI image analysis. InInternational Conference on Ubiquitous Computing and Multimedia Applications 2011 Apr 13 (pp. 307-314). Springer, Berlin, Heidelberg. 